\title{
Evaluation of regional myocardial perfusion in methamphetamine abusers using real-time myocardial contrast echocardiography
}

\author{
Xiao-Zhi Zheng ${ }^{1 *}$, Yun-Yan Shi ${ }^{*}$, Ke-Qi Chen', Xiao-Ling Qiao ${ }^{1}$, Lian-You Wang ${ }^{2}$ \\ * the authors shared the first authorship \\ ${ }^{1}$ Department of Ultrasound, Yancheng Institute of Clinical Medicine (The First People's Hospital of Yancheng), \\ Xuzhou Medical University, ${ }^{2}$ Hospital of Fangqiang Forced Quarantine and Drug Rehabilitation Center, Yancheng, \\ Jiangsu Province, P.R. China
}

\begin{abstract}
Aims: To evaluate the feasibility of assessing regional myocardial perfusion using real-time myocardial contrast echocardiography (MCE) at rest for detecting coronary microcirculation abnormalities in methamphetamine abusers. Material and methods: Twenty-two male methamphetamine abusers (11 without chest pain, 11 with chest pain), free of ascertained coronary artery disease, were enrolled in this study. A control group of 22 age-matched male healthy participants was studied for comparison. Standard 2D, flow and tissue Doppler echo with measurements of cardiac morphologic and functional indicators, MCE with measurements of regional myocardial perfusion were performed, respectively. Results: Compared to healthy participants, methamphetamine abusers had higher blood pressure, greater left ventricular mass index and more impaired diastolic function, with preserved cardiac sizes and systolic function. Methamphetamine abusers with chest pain had a faster heart rate than those without chest pain and healthy participants. MCE in methamphetamine abusers, especially with chest pain, had significant longer contrast agent arrival times, less functional capillary blood volumes, slower microvascular flow velocities and less myocardial perfusion than healthy participants $(\mathrm{p}<0.05)$. Moreover, along with the increases of dosage and duration of use (from group A to group C, group A: 1-2 g/day, $<2$ years; group B: 2-3 g/day, 2-5 years; group C: $>3 \mathrm{~g} / \mathrm{day},>5$ years) the reductions in the myocardial perfusion indices were more significant $(\mathrm{p}<0.01)$. The cutoff value with $5.1 \mathrm{~dB}^{2} / \mathrm{s}$ of the myocardial perfusion at the left ventricular apex had a sensitivity of $87.5 \%$, specificity of $75.2 \%$ and accuracy of $81.9 \%$ for differentiating methamphetamine abusers from normal subjects. Conclusions: Real-time MCE can effectively detect coronary microcirculation abnormalities in methamphetamine abusers at rest and myocardial perfusion is significantly reduced in methamphetamine abusers. This finding may be involved in the occurrence and development of cardiac damage.
\end{abstract}

Keywords: methamphetamine; chest pain; myocardial perfusion; myocardial contrast echocardiography

\section{Introduction}

Myocardial ischemia is one of the common injuries of methamphetamine use. The mechanism is related to

Received 12.08.2018 Accepted 07.11.2018

Med Ultrason

2019, Vol. 21, No 1, 56-61

Corresponding author: Xiao-Zhi Zheng

Department of Ultrasound, Yancheng Institute of Clinical Medicine (The First People's Hospital of Yancheng), Xuzhou Medical

University, 166 West Yulong Road,

Yancheng 224005, Jiangsu Province, P.R. China

Phone: +860515 88508717

Fax: +86051588500299

E-mail: zxzycyy1232@163.com the spasm (vasoconstriction) of the normal or atherosclerotic coronary arteries associated with increased platelet aggregation, blood pressure and heart rate $[1,2]$. Previous clinical research on methamphetamine-induced myocardial ischemia focused on large epicardial coronary arteries using invasive cardiac catheterization $[3,4]$ but studies concerning the coronary microcirculation abnormalities are lacking.

Real time myocardial contrast echocardiography (MCE) is a recently developed technique that utilizes low mechanical index to minimize microbubble destruction and permits quantitative measurements of myocardial perfusion from the immediate post-destruction frame of real-time perfusion according to time $v s$. backgroundsubtracted acoustic intensity plots $[5,6]$. This measure- 
ment enables the direct assessment of global and regional myocardial structure, the degree and adequacy of microvascular perfusion as well as the presence of wall motion abnormalities [7,8]. Therefore, MCE is regarded as an ideal tool for the detection of coronary artery stenosis, assessment of myocardial viability and correct triaging of patients to revascularization at rest or/and stress [8-10]. However, there are few data in the literature regarding the role of this technique for assessing regional myocardial perfusion in methamphetamine abusers.

We tested the hypothesis that real time MCE at rest could effectively detect the changes of myocardial perfusion in methamphetamine abusers, comparing the results with healthy participants.

\section{Material and methods}

\section{Study population}

Our study was approved by the Human Research Ethics Committee of Fangqiang Forced Quarantine and Drug Rehabilitation Center of the Jiangsu Province and free informed consent was obtained from all participants. A detailed cardiac history was collected at the recruitment from more than 2000 male adults of different methamphetamine exposure conditions (dosage: from $0.5 \mathrm{~g}$ to $6 \mathrm{~g} / \mathrm{d}$; duration: from 2 months to 10 years), including cardiovascular risk factors, clinical history or physical and echocardiographic exams. Exclusion criteria were: ascertained coronary artery disease and previous myocardial infarction, primary cardiomyopathies, congenital heart disease, arterial hypertension, hyperlipidemia, diabetes, previous 2D echocardiographic wall motion abnormalities, rhythm other than sinus, prosthetic valves or pacemakers, echocardiographic ejection fraction (EF) $<50 \%$, more than mild valvular stenosis or regurgitation, assumption of cardiac medications and standard echo images of inadequate quality. Finally, the study population consisted of 22 participants (19-51 years old, median age 37 years): 11 methamphetamine abusers without chest pain and 11 with chest pain. According to the dosages and durations of methamphetamine use the participants were also divided into three groups: group A, intake 1-2 $\mathrm{g} /$ day, $<2$ years $(\mathrm{n}=7)$; group $\mathrm{B}$, intake 2-3 g/day, 2-5 years $(n=7)$; and group $C$, intake $>3 \mathrm{~g} / \mathrm{d},>5$ years $(n=8)$. A control group of 22 age-matched male healthy participants (19-50 years old, median age 26 years) was used for comparison. All methamphetamine abusers underwent coronary angiography (digital subtraction angiography or computed tomography). Meanwhile, the electrocardiographic (ECG) findings (ST-T changes, sinoatrial block, atrioventricular block, complete right or left bundle branch block, premature beat, ectopic tachycardia, low voltage, or abnormal Q wave) and myocardial enzymes (troponin, creatine kinase, glutamate transaminase, lactate dehydrogenase, etc.) were recorded.

\section{Standard 2D, flow and tissue Doppler echo examination}

The echocardiographic study was performed in the left lateral decubitus position and with ECG recorded simultaneously. The echocardiographic data were acquired with a commercially available ultrasound system (Vivid E9, GE Healthcare, Milwaukee, WI) equipped with an M5S single-crystal matrix array transducer (1.7-3.3 $\mathrm{MHz}$ ). Left atrial end-systolic anteroposterior diameter (LA), left ventricular end-diastolic anteroposterior diameter (LV), right atrial end-systolic transverse diameter (RA), right ventricular end-diastolic anteroposterior diameter (RV), tricuspid annular plane systolic excursion (TAPSE), left ventricular ejection fraction (LVEF) and left ventricular mass index (LVM index) were obtained by real time 2D echo method. Flow Doppler sample was placed at the tip level of the mitral leaflets form the apical 4-chamber view. The peak early diastolic transmitral filling velocities (E) were measured. Tissue Doppler sample was placed at the septal mitral annulus form the apical 4-chamber view. The peak early diastolic septal tissue velocities (E') were measured and the ratio of E/E' were calculated.

\section{Real time $\mathrm{MCE}$}

A commercially available second-generation contrast agent, SonoVue ${ }^{\circledR}$ phospholipid-shell sulfur hexafluoride microbubbles (Bracco, Milan, Italy) was used. A total of $59 \mathrm{mg}$ of Sonovue ${ }^{\circledR}$ was diluted in $5 \mathrm{~mL}$ of saline according to the manufacturer's protocol. At the beginning $1.5 \mathrm{~mL}$ of this solution was administered intravenously at an infusion rate of about $0.5 \mathrm{~mL} / \mathrm{s}$ in order to gain a visible ventricular opacification and myocardial enhancement, quickly followed by the administration of $1 \mathrm{~mL}$ at an infusion rate of about $1 \mathrm{~mL} / \mathrm{min}$ to assure optimal myocardial enhancement.

MCE data were acquired with a commercially available ultrasound system (Mindray M9, Mindray Medical Systems, Shenzhen, China) equipped with a 1-5 MHz C5-1s transducer, using a multi-pulse contrast-specific imaging protocol (power modulation imaging) combined with pulse inversion power Doppler. Examination was performed from the apical four-chamber view with septum focused in the middle of the window. Gain, depth, transmit focus, and post-processing were optimized at the beginning of the study and held constant throughout. The optimal balance between myocardial contrast enhancement and attenuation in our setting was achieved at a very low mechanical index of 0.067 (acoustic power 4.8\%, thermal index of soft tissue, 0.0 ). The replenishment of 


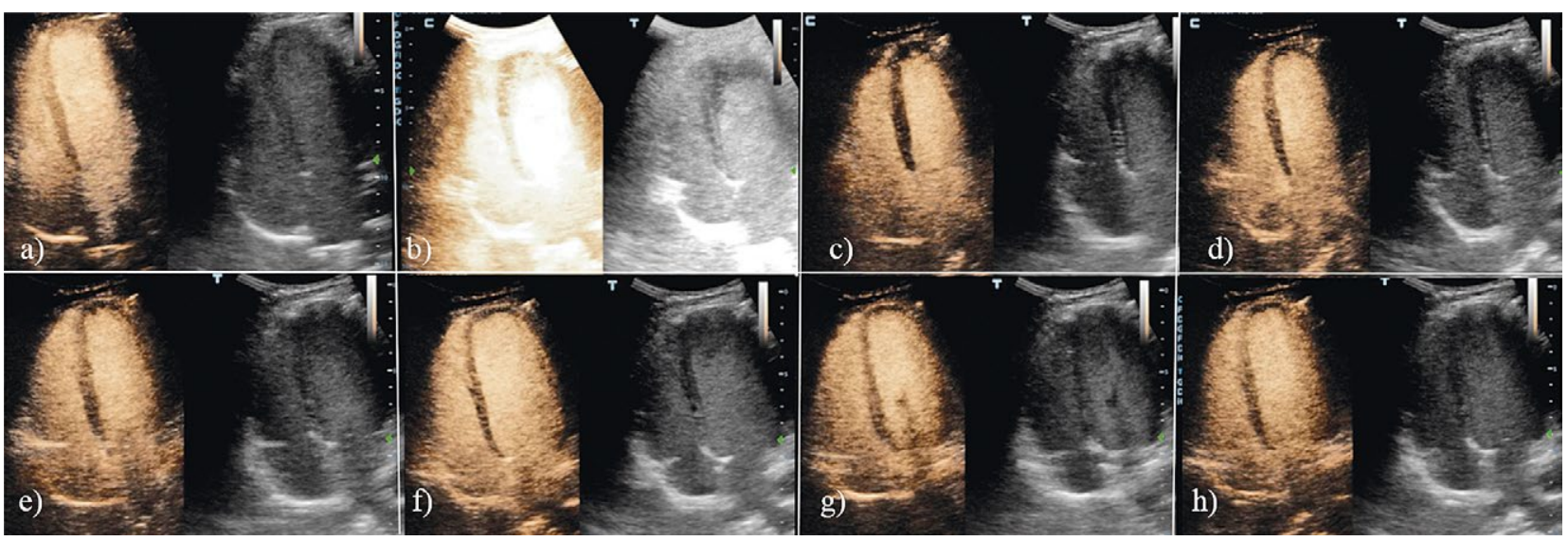

Fig 1. The myocardial contrast echocardiography obtained from a healthy participant at various time intervals: a) -1s (1s before the destructive pulse sequence) b) destructive pulse sequence (denoted as T0) and (b to h) at 3,5,10,15,20,30 s after the destructive pulse sequence, respectively.

myocardial Sonovue ${ }^{\circledR}$ at consecutive pulsing intervals after a destructive pulse with an MI of 1.352 (acoustic power $96.6 \%$, thermal index of soft tissue 0.3 ) was recorded; only end-systolic frames were used for analysis.

Image analysis was performed off-line by two experienced operators, according to established methods $[6,11]$. Briefly, a large region-of-interest was placed over the midventricular septum and left ventricular apex of twenty to forty consecutive end-systolic images beginning with the immediate post-destruction frame, respectively (fig 1). Time $v s$ background-subtracted acoustic intensity plots were then generated and fit to the exponential function $y=A\left(1-e^{\beta t}\right)$, where $y$ is the acoustic intensity at a time of $t, A$ is the plateau where acoustic intensity represents myocardial blood volume, and $\beta$ represents the mean microbubble flux rate through the microcirculation (referred to as microvascular flow velocity). Data are reported as the average of at least three separate microbubble destruction-refill time $v s$ acoustic intensity plots. Myocardial perfusion was calculated as the product of myocardial blood volume (A) and microvascular flow velocity $(\beta)$. In our study, $A$ and $\beta$ were the peak intensity (PI) and ascending slope (AS) derived from microbubble destruction-refill time $v s$ acoustic intensity plots, respectively (fig 2). In addition, the microbubble arrival times (ATs) were recorded.

Intraobserver and interobserver variability for measurements of $A$ and $\beta$ parameters were obtained by repeated, blinded analysis of 20 randomly selected patients after a minimum time interval of 2 weeks.

\section{Statistical analysis}

Results are expressed as mean \pm standard deviation. The differences between two groups were tested using unpaired samples t-test. A receiver operating characteristic curve (ROC) analysis of the myocardial perfusion was used to differentiate methamphetamine abusers from normal subjects, determine the optimal cut-off points and validity parameters. Bland \& Altman was used to measure the inter/intra observer variability. A value of $p<0.05$ was considered statistically significant. All statistical analysis was performed with SPSS version 16 software for Windows (SPSS Inc, Chicago, IL).

\section{Results}

Coronary angiography revealed unobstructed arteries in all methamphetamine abusers. All of them had normal blood glucose and lipid concentrations. The echocardiographic measurements were successfully completed in all subjects.

As shown in Table I, SBP, DBP, LVM index and E/E' were significantly greater in methamphetamine abusers with/without chest pain comparing with healthy participants $(p<0.05)$. HR was greater in methamphetamine abusers with chest pain than in those without chest pain

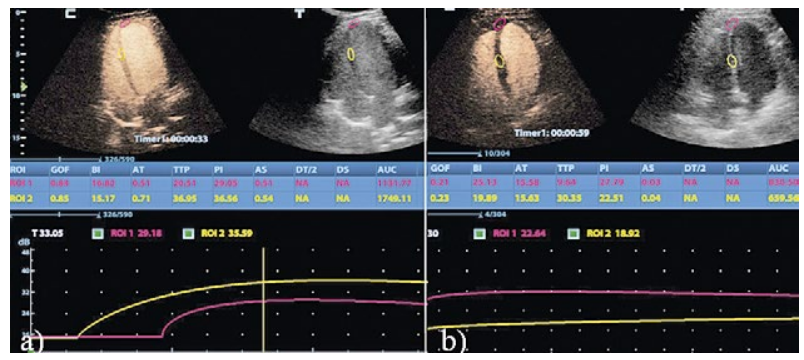

Fig 2. A typical time-intensity plot obtained from a healthy participant (a) and a methamphetamine abuser (b) (exposure condition, $2 \mathrm{~g}$ /day for 4 years). Note that the arrival time (AT) in this methamphetamine abuser appears significantly prolonged, the rate of bubble replenishment (AS) significantly reduced, and the maximum video intensity (PI) moderately reduced. 
and healthy participants. ECG traces and myocardial enzymes levels were abnormal in methamphetamine abusers with chest pain and normal in those without chest pain and healthy participants. Age, LV, LA, RV, RA, LVEF and TAPSE were not significantly different among methamphetamine abusers with/without chest pain and healthy participants $(\mathrm{p}>0.05)$.

In table II are detailed the differences of MCE indices among healthy participants, methamphetamine abusers with chest pain and those without chest pain. Compared to healthy participants, methamphetamine abusers had significant longer contrast agent arrival times (ATs), less functional capillary blood volumes (PIs), slower microvascular flow velocities (ASs) and less myocardial perfusion $(\mathrm{PI} \times \mathrm{AS})$. Moreover, methamphetamine abusers with chest pain also had longer ATs, less functional capillary blood volumes (PIs), slower microvascular flow velocities (ASs) and less myocardial perfusion $(\mathrm{PI} \times \mathrm{AS})$ than those without chest pain.
Table III and figure 3 showed the changes of MCE indices under different methamphetamine exposure conditions. From group A to group C, contrast agent arrival times (ATs) extended sequentially and the functional capillary blood volume (PI), microvascular flow velocity (AS) and myocardial perfusion (PI $\times$ AS $)$ decreased successively.

The area under the curve (AUC) of the myocardial perfusion at the left ventricular apex was 0.92 (0.84-1.00) at a cutoff value with $5.1 \mathrm{~dB}^{2} / \mathrm{s}$ with sensitivity of $87.5 \%$, specificity of $75.2 \%$ and accuracy of $81.9 \%$ for differentiating methamphetamine abusers from normal subjects. The AUC of the myocardial perfusion at midventricular septum was 0.84 (0.71-0.97) at a cutoff value with 4.3 $\mathrm{dB}^{2} / \mathrm{s}$ with sensitivity of $87.5 \%$, specificity of $68.9 \%$ and accuracy of $77.3 \%$ for differentiating methamphetamine abusers from normal subjects.

Interobserver and intraobserver agreement for measurement of the MCE indices (ATs, PIs and ASs) were

Table I. Characteristics of healthy participants and methamphetamine abusers (MBs) with/without chest pain

\begin{tabular}{llll}
\hline Variables & Healthy participants & MBs without chest pain $(\mathbf{n}=\mathbf{1 1})$ & MBs with chest pain $(\mathbf{n}=\mathbf{1 1})$ \\
\hline Age, $\mathrm{y}$ & $38.2 \pm 8.4$ & $37.9 \pm 8.1$ & $38.5 \pm 8.3$ \\
$\mathrm{HR}$, beats/min & $73.34 \pm 8.47$ & $75.62 \pm 9.34$ & $96.54 \pm 12.34^{*}$ \\
SBP, mm Hg & $112.45 \pm 11.37$ & $126.88 \pm 19.78^{*}$ & $139.74 \pm 21.76^{*}$ \\
DBP, mm Hg & $63.77 \pm 16.56$ & $74.56 \pm 18.39^{*}$ & $93.96 \pm 20.17^{*}$ \\
ECG findings & Normal & Normal & Abnormal \\
Myocardial enzymes & Normal & Normal & Abnormal \\
RV, mm & $18.26 \pm 2.18$ & $18.89 \pm 3.37$ & $19.65 \pm 3.82$ \\
RA, mm & $30.66 \pm 4.78$ & $31.65 \pm 4.90$ & $32.76 \pm 4.86$ \\
LV, mm & $40.34 \pm 4.44$ & $41.67 \pm 4.65$ & $42.89 \pm 4.43$ \\
LA, mm & $26.78 \pm 5.29$ & $30.67 \pm 6.43$ & $31.62 \pm 5.10$ \\
LVEF(\%) & $68.56 \pm 9.13$ & $66.54 \pm 8.59$ & $69.67 \pm 9.43$ \\
LVM index, g/m 2 & $87.52 \pm 15.49$ & $109.93 \pm 21.57^{*}$ & $112.78 \pm 21.66^{*}$ \\
E/E & $4.98 \pm 1.39$ & $6.92 \pm 1.93^{*}$ & $7.08 \pm 2.16^{*}$ \\
TAPSE & $18.59 \pm 6.67$ & $17.95 \pm 7.56$ & $17.83 \pm 7.95$ \\
\hline
\end{tabular}

* $\mathrm{p}<0.05$; Data are expressed as mean \pm standard deviation. DBP - diastolic blood pressure; E/E' - ratio of peak early diastolic transmitral filling velocity (E) and peak early diastolic septal mitral annulus tissue velocity (E'); HR - heart rate; LA - left atrial end-systolic anteroposterior diameter; LV - left ventricular end-diastolic anteroposterior diameter; RA - right atrial end-systolic transverse diameter; RV - right ventricular end-diastolic anteroposterior diameter; SBP - systolic blood pressure; TAPSE - tricuspid annular plane systolic excursion.

Table II. Comparison of regional myocardial perfusion in healthy participants and methamphetamine abusers (MBs)

\begin{tabular}{llll}
\hline Variables & Healthy participants & MBs without chest pain $(\mathbf{n}=11)$ & MBs with chest pain (n=11) \\
\hline Apex & $1.66 \pm 0.87$ & $6.82 \pm 2.59^{*}$ & $17.54 \pm 6.75^{* * \dagger}$ \\
$\mathrm{AT}, \mathrm{s}$ & $43.64 \pm 5.80$ & $27.02 \pm 3.53^{*}$ & $17.94 \pm 1.48^{* * \dagger}$ \\
$\mathrm{PI}, \mathrm{dB}$ & $0.83 \pm 0.22$ & $0.25 \pm 0.17^{*}$ & $0.13 \pm 0.05^{* * \dagger}$ \\
$\mathrm{AS}, \mathrm{dB} / \mathrm{s}$ & $37.98 \pm 11.31$ & $7.37 \pm 4.01^{*}$ & $2.33 \pm 0.94^{* * \dagger}$ \\
$\mathrm{PI} \times \mathrm{AS}, \mathrm{dB} / \mathrm{s}$ & & $19.33 \pm 7.78^{* * \dagger}$ \\
interventricular septum & & $7.09 \pm 2.88^{*}$ & $15.66 \pm 1.11^{* * \dagger}$ \\
$\mathrm{AT}, \mathrm{s}$ & $1.83 \pm 0.92$ & $23.76 \pm 5.42^{*}$ & $0.11 \pm 0.06^{* * \dagger}$ \\
$\mathrm{PI}, \mathrm{dB}$ & $39.33 \pm 6.02$ & $0.18 \pm 0.28^{*}$ & $1.72 \pm 0.63^{* * \dagger}$ \\
$\mathrm{AS}, \mathrm{dB} / \mathrm{s}$ & $0.56 \pm 0.15$ & $5.22 \pm 10.53^{*}$ & $2.17 \pm 9.85$ \\
$\mathrm{PI} \times \mathrm{AS}, \mathrm{dB} / \mathrm{s}$ & 22.85 &
\end{tabular}

Data are expressed as mean \pm standard deviation. PI - peak intensity; AS - ascending slope; AT - arrival time. *p<0.05; ** $<0.01$, values are different from healthy participants; $\uparrow \mathrm{p}<0.05 ; \dagger \uparrow \mathrm{p}<0.01$ values are different from methamphetamine abusers without chest pain 
Table III. Comparison of regional myocardial perfusion among methamphetamine abusers of different exposure conditions (A: 1-2g/ day, <2 years; B: 2-3g/day, 2-5 years; C: $>3 \mathrm{~g}$ /day, $>5$ years)

\begin{tabular}{llll}
\hline Variables & Group A (n=7) & Group B (n=7) & Group C (n=8) \\
Apex & & & $26.33 \pm 9.27^{* \dagger}$ \\
AT, s & $6.77 \pm 2.43$ & $13.89 \pm 5.58^{*}$ & $8.57 \pm 1.55^{* \dagger}$ \\
PI, dB & $29.34 \pm 5.66$ & $17.02 \pm 4.29^{*}$ & $0.13 \pm 0.06^{* \dagger}$ \\
AS, dB/s & $0.31 \pm 0.17$ & $0.22 \pm 0.07^{*}$ & $1.14 \pm 0.39^{* \dagger}$ \\
PI $\times$ AS, dB2/s & $9.12 \pm 2.37$ & $3.76 \pm 1.22^{*}$ & $27.18 \pm 9.55^{* \dagger}$ \\
interventricular septum & & & $7.43 \pm 1.81^{* \dagger}$ \\
AT, s & $7.06 \pm 2.53$ & $15.44 \pm 7.94^{*}$ & $0.12 \pm 0.05^{* \dagger}$ \\
PI, dB & $26.27 \pm 6.33$ & $13.89 \pm 4.62^{*}$ & $0.89 \pm 0.24^{* \dagger}$ \\
AS, dB/s & $0.26 \pm 0.16$ & $0.17 \pm 0.07^{*}$ & $2.39 \pm 0.92^{*}$ \\
PI $\times \mathrm{AS}, \mathrm{dB} 2 / \mathrm{s}$ & $6.86 \pm 2.12$ & & \\
\hline
\end{tabular}

Data are expressed as mean \pm standard deviation. PI - peak intensity; AS - ascending slope; AT - arrival time. ${ }^{*} \mathrm{p}<0.01$, values are different from the group $\mathrm{A} ; \uparrow \mathrm{p}<0.01$, values are different from the group $\mathrm{B}$

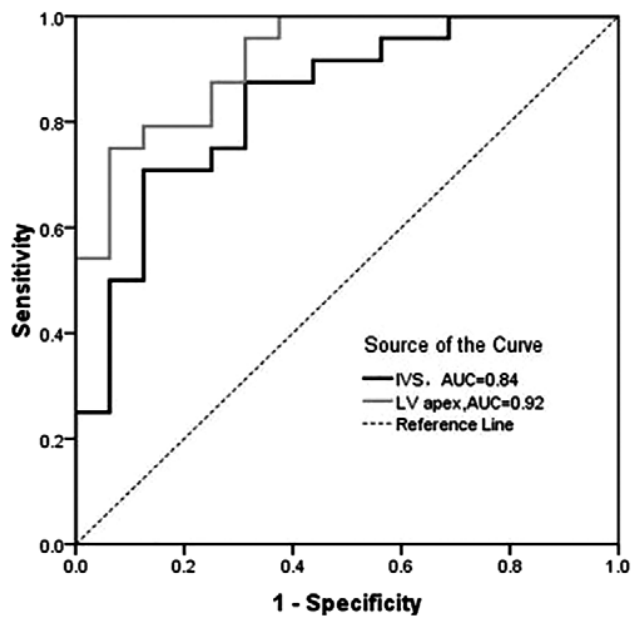

Fig 3. The performance of the myocardial perfusion for differentiating methamphetamine abusers from normal subjects using receiver operating characteristic curve analysis. IVS - interventricular septum; LV apex - Left ventricular apex.

all very good: intraclass correlation coefficients were all $>0.98$ for interobserver and intraobserver variability.

\section{Discussions}

This is the first study on the feasibility of assessing regional myocardial perfusion in chronic methamphetamine addicts recruited from drug rehabilitation center using real-time MCE. This study showed that real-time MCE can effectively detect the changes of regional myocardial perfusion in methamphetamine abusers at rest and that the regional myocardial perfusion was significantly reduced in methamphetamine abusers. The technique had a good performance on differentiation of methamphetamine abusers from normal subjects. To the best of our knowledge, real-time MCE had never been used before for serial assessment in this clinical setting.
During the last few years, second generation contrast agents (e.g., SonoVue ${ }^{\circledR}$ phospholipid-shell sulfur hexafluoride microbubbles) have become commercially available in China. Meanwhile, the improved image quality in echocardiography, combined with new ultrasound technical developments (e.g., power modulation imaging and pulse inversion power Doppler) made it possible to obtain echocardiographic images of myocardial perfusion. Coronary microcirculation contains $80 \%$ of the total myocardial blood volume and regulates nutrient exchange in cardiac myocytes [12]. Using echogenic gasfilled microspheres with similar size and rheologic properties to red blood cells, the destruction-refill kinetic data permit repeated quantitative measures of microvascular flow velocity and capillary blood volume. Now, MCE is technically matured and has gained wide use in the clinical diagnosis and laboratory researches. In this study, we employed a commercially available ultrasound system, Mindray M9, equipped with a $1-5 \mathrm{MHz}$ C5-1s transducer, using a multi-pulse contrast-specific imaging protocol (power modulation imaging), combined with pulse inversion power Doppler, utilizing contrast agent characters with both $2^{\text {nd }}$ harmonic and non-linear fundamental signal to get improved signal-to-noise ratio for better diagnostic details and longer contrast agent duration for better observation.

The main findings of our study are that methamphetamine abusers, especially ones with chest pain, had significant longer contrast agent arrival times, less functional capillary blood volumes, slower microvascular flow velocities and less myocardial perfusion than healthy participants. Moreover, along with the increase of dosage and duration of use, the reductions in these myocardial perfusion indices were more significant. These findings suggest that methamphetamine induces constriction at the level of the coronary microcirculation and the major site of methamphetamine's action is probably at the most 
distal coronary microvessels and capillary networks. These findings provided direct evidence for our previous studies in which methamphetamine has been shown to decrease coronary sinus flow and myocardial function $[13,14]$.

Our data are consistent with Gurudevan et al in an earlier MCE study [6] in 10 healthy young men, in which the authors demonstrated that low-dose cocaine challenge evokes a sizeable decrease in myocardial perfusion. However, in our study we found that the predominant effect of methamphetamine was to decrease both myocardial capillary blood volume and microvascular flow velocity, while in Gurudevan et al earlier study cocaine decreased myocardial capillary blood volume rather than microvascular flow velocity. The different findings in our study may be due to the different populations studied (chronic methamphetamine-addicted patients, not healthy young men), half of them with previous chest pain and ischemic ECG changes and different methamphetamine exposure conditions.

Our study had several limitations that should be considered. First, methamphetamine abusers had greater blood pressure and more impaired LV diastolic function than healthy participants. An increased LV pressure from diastolic dysfunction theoretically could contribute to our results by causing extravascular compression of coronary microvessels. However, we did not measure LV end-diastolic pressure. Secondly, the repeatability of measurement of ascending slope from microbubble destruction-refill time vs acoustic intensity plots was always poor. New quantitative measurements methods of myocardial perfusion are needed. Thirdly, the sample size of the study was so small that grouping based on without/with chest pain and grouping based on different exposure conditions was difficult. A series of studies based on larger populations would be more persuasive. Finally, we assessed LV regional myocardial perfusion only at rest. It would be interesting to expand this study to $\mathrm{RV}$ myocardial segments at rest or/and at stress.

\section{Conclusions}

Our study demonstrated that regional myocardial perfusion is significantly reduced in methamphetamine abusers, especially in those with chest pain. Although it had some limitations, as mentioned above, this study still enriches the understanding of the pathogenesis of chest pain in methamphetamine abusers and still holds considerable clinical promise for monitoring, predicting and follow-up of the myocardial ischemia and cardiac toxicity of methamphetamine as well as the effects of detoxification treatment.
Acknowledgements: The authors gratefully acknowledge the technical assistance of Xia EH, Huang XQ and Fan GX from the Department of Ultrasound, The First People's Hospital of Yancheng, Jiangsu Province, P.R.China.

\section{Conflict of interest: none}

\section{References}

1. Ciccarone D. Stimulant abuse: pharmacology, cocaine, methamphetamine, treatment, attempts at pharmacotherapy. Prim Care 2011;38:41-58.

2. Rezkalla SH, Kloner RA. Cocaine-induced acute myocardial infarction. Clin Med Res 2007;5:172-176.

3. Kanwar M, Gill N. Spontaneous multivessel coronary artery dissection. J Invasive Cardiol 2010;22:E5-E6.

4. Wijetunga M, Seto T, Lindsay J, Schatz I. Crystal methamphetamine-associated cardiomyopathy: tip of the iceberg? J Toxicol Clin Toxicol 2003;41:981-986.

5. Fernandes DR, Tsutsui JM, Bocchi EA, et al. Qualitative and quantitative real time myocardial contrast echocardiography for detecting hibernating myocardium. Echocardiography 2011;28:342-349.

6. Gurudevan SV, Nelson MD, Rader F, et al. Cocaine-induced vasoconstriction in the human coronary microcirculation: new evidence from myocardial contrast echocardiography. Circulation 2013;128:598-604.

7. Ito H. Myocardial contrast echocardiography after myocardial infarction. Curr Cardiol Rep 2012;14:350-358.

8. Li DY, Hao J, Xia Y, et al. Clinical usefulness of low-dose dobutamine stress real-time myocardial contrast echocardiography for detection of viable myocardium. J Clin Ultrasound 2012;40:272-279.

9. Li DY, Liang $\mathrm{L}, \mathrm{Xu} \mathrm{TD}$, et al. The value of quantitative real-time myocardial contrast echocardiography for detection of angiographically significant coronary artery disease. Clin Cardiol 2013;36:468-474.

10. Porter TR, Xie F. Myocardial perfusion imaging with contrast ultrasound. JACC Cardiovasc Imaging 2010;3:176187.

11. Wei K, Jayaweera AR, Firoozan S, Linka A, Skyba DM, Kaul S. Quantification of myocardial blood flow with ultrasound-induced destruction of microbubbles administered as a constant venous infusion. Circulation 1998;97:473-483.

12. Kassab GS, Lin DH, Fung YC. Morphometry of pig coronary venous system. Am J Physiol 1994;267:H2100H2113.

13. Wei GL, Zheng XZ, Chen KQ, Shi YY, Wang LY, Tan $\mathrm{XY}$. Coronary sinus flow is reduced in methamphetamine abusers:a transthoracic echocardiographic study. Int J Cardiovasc Imaging 2018;34:1889-1894.

14. Zhang LJ, Chen KQ, Shi YY, Qiao XL, Wang LY, Zheng $\mathrm{XZ}$. Findings on 3D speckle tracking echocardiography in asymptomatic methamphetamine abusers. Int J Cardiovasc Imaging 2018;34:1589-1593. 